\title{
A novel de novo $D D X 3 X$ missense variant in a female with brachycephaly and intellectual disability: a case report
}

\author{
Giada Moresco ${ }^{1}$, Jole Costanza', Carlo Santaniello', Ornella Rondinone ${ }^{1}$, Federico Grilli², Elisabetta Prada², \\ Simona Orcesi ${ }^{3,4}$, llaria Coro ${ }^{2}$, Anna Pichiecchio ${ }^{4,5}$, Paola Marchisio ${ }^{2,6}$, Monica Miozzo ${ }^{1,7}$, Laura Fontana ${ }^{1,7^{*}}$ and \\ Donatella Milani ${ }^{2}$
}

\begin{abstract}
Background: De novo pathogenic variants in the DDX3X gene are reported to account for $1-3 \%$ of unexplained intellectual disability (ID) in females, leading to the rare disease known as DDX3X syndrome (MRXSSB, OMIM \#300958). Besides ID, these patients manifest a variable clinical presentation, which includes neurological and behavioral defects, and abnormal brain MRIs.

Case presentation: We report a 10-year-old girl affected by delayed psychomotor development, delayed myelination, and polymicrogyria (PMG). We identified a novel de novo missense mutation in the DDX3X gene (c.625C > G) by whole exome sequencing (WES). The DDX3X gene encodes a DEAD-box ATP-dependent RNAhelicase broadly implicated in gene expression through regulation of mRNA metabolism. The identified mutation is located just upstream the helicase domain and is suggested to impair the protein activity, thus resulting in the altered translation of DDX3X-dependent mRNAs. The proband, presenting with the typical PMG phenotype related to the syndrome, does not show other clinical signs frequently reported in presence of missense DDX3X mutations that are associated with a most severe clinical presentation. In addition, she has brachycephaly, never described in female DDX3X patients, and macroglossia, that has never been associated with the syndrome.
\end{abstract}

Conclusions: This case expands the knowledge of DDX3X pathogenic variants and the associated DDX3X syndrome phenotypic spectrum.

Keywords: DDX3X, Rare disease, Intellectual disability, Polymicrogyria, Brachycephaly, Macroglossia, Case report

\section{Background}

About 1-3\% of females with unexplained intellectual disability (ID) carry de novo pathogenic variants in the X-linked $D D X 3 X$ gene (Xp11.4) [1]. Up to about 300 cases have been identified [2], both females with Xlinked dominant inheritance, and very few males showing an X-linked recessive pattern of inheritance [1].

\footnotetext{
* Correspondence: laura.fontana@unimi.it

${ }^{1}$ Research Laboratories Coordination Unit, Fondazione IRCCS Ca' Granda Ospedale Maggiore Policlinico, Milan, Italy

${ }^{7}$ Department of Health Science, Università degli Studi di Milano, Milan, Italy Full list of author information is available at the end of the article
}

Patients with the rare $D D X 3 X$ syndrome (MRXSSB, OMIM \#300958) show a variable clinical presentation with different degrees of ID and/or developmental delay, neurological and behavioral defects, including microcephaly, hypotonia, epilepsy, movement disorders, autism spectrum disorder and aggressiveness. Brain malformations are also reported, and include corpus callosum hypoplasia, ventricular enlargement, and polymicrogyria (PMG). Additional clinical features, among which facial dysmorphisms and sensory deficits, may also be present. 
To date, all reported pathogenic $D D X 3 X$ variants identified in affected females are de novo loss of function, leading to haploinsufficiency and, probably, to embryonic lethality in males. Most of $D D X 3 X$ mutations reported in males are maternally inherited missense variants. In these families, males showed borderline to severe ID and carrier females were unaffected and expressed a DDX3X protein retaining a partial functionality [1]. Several hypotheses have been evaluated to explain the gender-specific pathogenicity, including a skewing X-inactivation pattern, but no definitive conclusions have been drawn. Kellaris et al. [3] proposed that hypomorphic $D D X 3 X$ variants may be viable in hemizygous males and do not cause clinical phenotype in female carriers. However, few de novo $D D X 3 X$ mutations have been identified in males [4]. Although these male patients share many of the clinical features with affected females, some distinct clinical phenotypes can depend on the gender of the patient and on the pathogenicity of the variant. These findings suggest that the $D D X 3 X$ gene exerts dose-dependent and gender-specific effects in normal development and disease [5]. Indeed, Snijders Blok et al. [1] reported a family with recurrent miscarriages of unknown gender and a male viable pregnancy, terminated after the identification of severe congenital anomalies by ultrasound imaging. By whole exome sequencing (WES) a missense mutation in the $D D X 3 X$ gene was detected in the fetus, suggesting a potential germline mosaicism in the mother.

The $D D X 3 X$ gene encodes a DEAD-box ATPdependent RNA-helicase broadly implicated in gene expression through regulation of mRNA metabolism [6]. It acts as a translational regulator of target mRNAs with highly structured $5^{\prime}$ untranslated regions (UTRs) and it is involved in repeat-associated non-AUG translation [7]. DDX3X also takes part in stress response and stress granule assembly, innate immune signaling, mitotic chromosome segregation and it can also exert a role in tumorigenesis [8]. In addition, it is thought to be an essential factor in the RNA-interference pathway [9], and it is a key regulator of the $\mathrm{Wnt} / \beta$-catenin pathway [10].

Despite the ubiquitous expression of the DDX3X protein, high expression levels are detected in all the cortical layers of the embryonic brain, consistent with its crucial role in cortical development during neurogenesis [10] and, as a consequence, $D D X 3 X$ mutations are reported to impact on neuronal function [4]. In particular, defective neurite outgrowth and neural progenitor differentiation/migration could account for brain malformations and the consequent characteristic clinical phenotypes of the $D D X 3 X$ syndrome [5].

Here, we report a 10-year-old girl with delayed psychomotor development, delayed myelination, bilateral frontal PMG and thin body and splenium of the corpus callosum. WES allowed the identification of the novel de novo $D D X 3 X$ mutation c.625C > G (p.His209Asp), leading to a diagnosis of $D D X 3 X$ syndrome and expanding the number of $D D X 3 X$ pathogenic variants and their associated phenotypic spectrum.

\section{Case presentation}

The proband was referred to our laboratory by the pediatric geneticists of our Institution, Fondazione IRCC S Ca' Granda Ospedale Policlinico (Milan). Appropriate written informed consent was obtained from all family members.

Patient II-3 (Fig. 1A), who is currently 10 years old, is the third-born of healthy parents with a doubtful distant consanguinity (beyond the second generation). The two older siblings (II- 1 and II-2, of 16 and 14 years old respectively) are both healthy. Between the first and second pregnancy three miscarriages of unknown gender occurred, but no additional information is available. The family history is negative for genetic conditions.

The patient was born at $38+5$ gestational weeks, after a normal pregnancy, characterized by regular growth, morphology and fetal movements. She was born by cesarean delivery scheduled for previous cesarean section.

Clinical signs are reported in Table 1 . At birth, the child was $2740 \mathrm{~g}$ (25th centile), $49 \mathrm{~cm}$ long (50th centile), presented an occipitofrontal circumference of $34 \mathrm{~cm}$ (50th centile) and an Apgar score of 10-10. Absence of skin adnexa (eyelashes, eyebrows and nails) and difficult scarring of the umbilical stump are referred. In addition, a bilateral II-III toe syndactyly and a small left preauricular tag were evident. Ultrasounds revealed normal abdominal and cerebral morphology.

In the first months of life, a severe gastro-esophageal reflux with initial weight loss and sleep disturbance was reported, which improved after the introduction of proton-pump inhibitors therapy.

Growth was regular, but psychomotor development was severely delayed, with independent walking and babbling acquired towards 6 years of age.

Evaluation at 6 years old revealed the following craniofacial dysmorphisms: brachycephaly and a flattenedtriangular-asymmetrical face characterized by micrognathia, mild hypertelorism, wide and prominent nose, short philtrum, thin lips and macroglossia. She also presented a slight facial grimacing, large and anteverted ears and small left ear tag. Growth parameters were normal, with a weight of $16.5 \mathrm{~kg}$ (10-25th centiles), a height of $111 \mathrm{~cm}$ (50-75th centile), head circumference of $50.5 \mathrm{~cm}$ (25-50th centile).

Brain MRI performed at 6 months of age showed delayed myelination (improved at the second MRI performed at 2 years old), bilateral frontal PMG and thin 
A

I

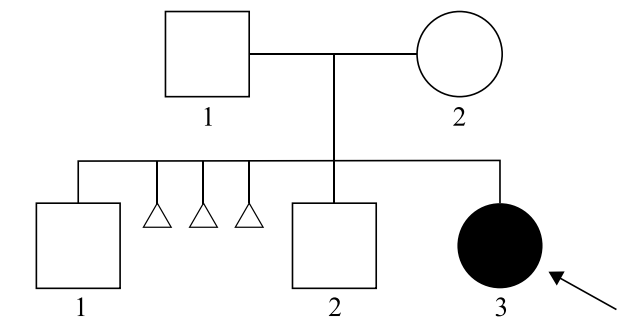

C

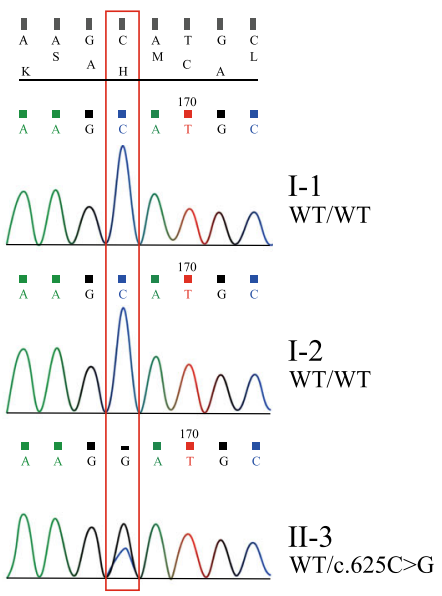

\section{B}

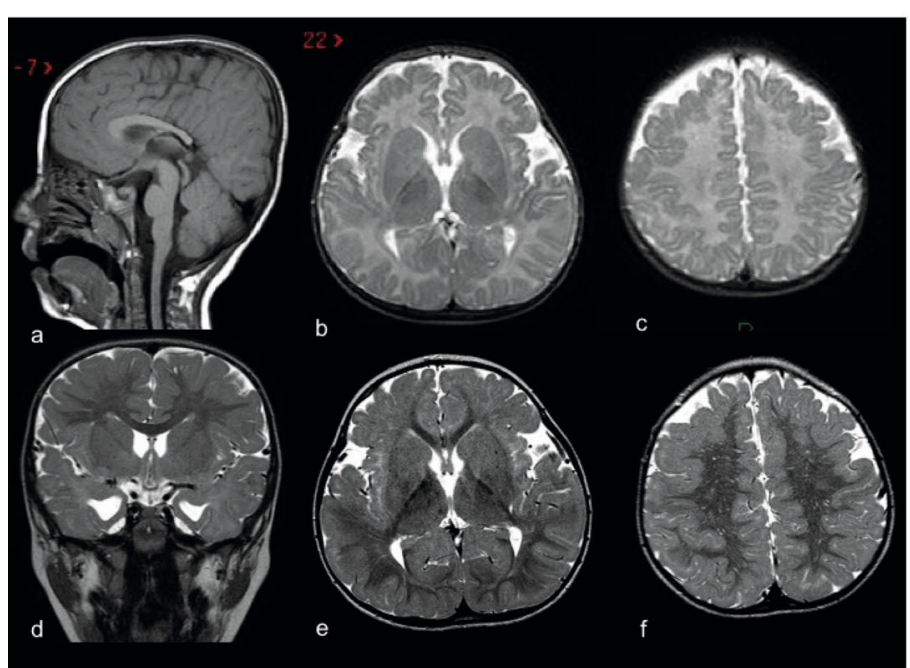

Fig. 1 A) Pedigree of the family. B) Brain MRI with sagittal T1-weighted sequence (a) and axial (b-c-e-f) and coronal (d) T2-weighted sequences, performed respectively at 6 months of age $(b-c)$ and 2 years old (a-d-e-f). A thin posterior corpus callosum is evident (a), involving the body, the isthmus and the splenium, with consequent dysmorphic temporal horns (d). A delayed myelination is evident at 6 months (b-c) as lack of diminution of signal intensity within the centrum semiovale, bilaterally (c) and in the corpus callosum (b); in the control MRI, myelination is complete (e-f), with evidence of numerous perivascular spaces within the white matter ( $f$ ). Frontal cortical thickening is evident, with irregularity of the cortical-white matter junction (c-f) in both hemispheres, consistent with PMG . C) Sequencing chromatograms of DNA samples from the proband and the parents. The position of the novel DDX3X variant identified is indicated by a red box. The proband is confirmed to be heterozygous for the DDX3X c.625C > G variant, while parents are wild-type

body and splenium of the corpus callosum (Fig. 1B). Electroencephalogram (EEG) showed sharp-wave anomalies on the rear right regions. She also presents hyperopia and divergent strabismus.

Karyotype, aCGH and mutational analysis of the ADGRG1 gene (associated to bilateral frontoparietal PMG resulted normal. An NGS panel for Rett syndrome (including the $M E C P 2, C D K L 5, F O X G 1, M E F 2 C$, SCN1A, UBE3A, PCDH19, STXBP1 genes) was also performed, and no pathogenic variants were identified.

Since no other clinical suspicions were hypothesized, the proband and her parents underwent WES on DNA extracted from peripheral blood leukocytes. Trio WES and variants interpretation according to the pedigree and the clinical features allowed the identification of the novel missense variant c.625C > G (p.His209Asp) in exon 7 of the $D D X 3 X$ gene. No other candidate variants were found, and the identified variant was confirmed by Sanger sequencing. The absence of the mutation in the healthy mother confirmed the hypothesis of a de novo inheritance (Fig. 1C). However, considering the occurrence of three miscarriages between the first and the second pregnancy, we cannot exclude the possibility that also the aborted fetuses could have carried the c.625C > $\mathrm{G}$ mutation as a consequence of a possible germline mosaicism in the mother. 
Table 1 Clinical features of II-3 compared to the characteristic clinical sings reported for the DDX3X syndrome and relative prevalence

\begin{tabular}{l} 
DDX3X syndrome clinical signs (relative \\
prevalence) \\
\hline Development \\
Developmental delay (106/106) [10] \\
Intellectual disability (106/106) [10] \\
Language delay (38/75) [10] \\
Growth \\
Failure to thrive (13/44) [11] \\
Short stature (1/6) [11] \\
Microcephaly (39/107) [11] \\
Brachycephaly (3/11) [11] \\
Neurologic/behavioral \\
Seizures (24/116) [10] \\
Hypotonia (66/116) [10] \\
Hypertonia/spasticity (9/78) [11] \\
Mixed hypo and hypertonia (31/93) [10] \\
Sleep disturbance (2/6) [11] \\
Movement disorders/spasticity in the legs (22/49) [11] \\
Behavior disorders/autism spectrum disorder/ \\
aggression (24/49) [11] \\
Hyperreflexia (9/78) [11] \\
Brain MRI \\
Polymicrogyria (11/89) [10] \\
Corpus callosum hypoplasia/agenesis (76/105) [11] \\
Ventricular enlargement (27/105) [11] \\
Key-hole shaped temporal horns (32/89) [10] \\
Colpocephaly (3/89) [10] \\
Delayed myelination/decreased cortical white \\
matter (50/89) [10] \\
Small pons (11/89) [10] \\
Senfior vermis (6/89) [10]
\end{tabular}

\section{Sensory}

Vision problems (strabismus, coloboma, astigmatism, nystagmus) (29/92) [10]

Hearing problems (11/114) [11]

\section{Facial dysmorphisms}

Short/down-slanting palpebral fissure length (2/6) [11]

Hypertelorism/telecanthus (6/36) [11]

Epicanthal folds (1/6) [11]

Elongated/flattened/triangular face (9/36) [11]

High/broad forehead (8/36) [11]

Wide nasal bridge/bulbous tip (9/36) [11]

Short/narrow nose, anteverted nares (11/36) [11]

Micrognathia (2/6) [11]
Clinical signs of II-3

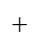

$+$

$+$

-

$-$

$+$

EEG

anomalies

$+$

$-$

$-$

$+$

$+$

$+$

+ (anterior)

$+$

$-$

-

$-$

$+$

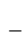

-

$-$

$+$

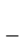


of information on the aborted fetuses and the impossibility to assess the presence of the germline mosaicism in the mother prevents us to confirm this hypothesis.

The novel c.625C > G variant involves the amino acidic position 209, which maps $2 \mathrm{bp}$ upstream the helicase ATP binding domain (Fig. 2). Most reported DDX3X mutations map within the two helicase domains impairing the protein helicase activity, with a dominant negative mechanism [10]. The novel His209Asp variant is close to the Val206Met, already reported in a patient with the $D D X 3 X$ syndrome [10]. Despite no functional tests have been performed to confirm the pathogenic effect of this novel mutation, according to in silico predictions and literature data on the close Val206Met and other missense mutations that fall within or in proximity of the DDX3X helicase domain [10], we hypothesize that also the His209Asp variant leads to an impaired helicase activity. Deficient DDX3X helicase activity results in the enzyme inability to release the bound RNA after ATP hydrolysis and the altered translation of DDX3Xdependent targets, in particular mRNAs containing highly structured $5^{\prime}$ UTRs and/or high GC content [12]. This leads to the sequestration of RNAs and RNA binding proteins, and the formation of aberrant ribonucleoprotein (RNP) granules containing newly synthetized proteins and/or stalled polysomes [10]. The defective translation of DDX3X-target transcripts has been proposed to lead to impaired neurogenesis, likely affecting embryonic cortical development [10]. This mechanism may also account for the more severe phenotypes associated with missense mutations, compared to nonsense and frameshift (presumed loss of function) lesions, that affect translation of some targets but do not induce granule formation, resulting in a less severe clinical presentation [10]. This pathomechanism may also apply to the His209Asp mutation here reported, as suggested, not only by the affected protein domain, but also by the PMG phenotype of the proband herein presented.
According to the recent studies on genotype-phenotype correlations in the $D D X 3 X$ syndrome and underlying pathogenetic mechanisms [10], patients with severe cerebral defects, including complete or partial absence of the corpus callosum and PMG (frequently associated to microcephaly), presented missense mutations or single amino acid deletion hotspot mutations, including the nearby Val206Met (Fig. 2). These mutations are also associated to more complex clinical presentations, including epilepsy, autism spectrum disorder, severe intellectual disability, and cardiovascular anomalies, not present in patients without PMG [10]. This evidence thus suggests that these variants are likely to lead to a more severe dominant negative phenotype, while loss of function mutations are associated to a milder spectrum of clinical traits with no signs of PMG. Despite the proband herein described presents with PMG, she does not show other clinical traits reported in most severe cases of $D D X 3 X$ syndrome, such as microcephaly, congenital cardiac defects and epilepsy (she only presents EEG anomalies) (Table 1). In addition, she shows reduced subcutaneous fat, already reported in two other patients $D D X 3 X$ syndrome patients [11], and she is the first female patient presenting with brachycephaly (which has so far been described only in few males [1]) (Table 1). She also has macroglossia, an additional clinical sign that has never been associated with the $D D X 3 X$ syndrome before (Table 1 ).

In conclusion, these distinctive clinical features, together with the presence of PMG, but no other signs associated to a severe phenotype, furtherly expand genotype-phenotype correlations of $D D X 3 X$ missense mutations.

This case report emphasizes the clinical utility of WES in ending the diagnostic odyssey of individuals with unexplained ID.

\section{Abbreviations}

DDX3X: DEAD-Box Helicase 3 X-Linked; ID: Intellectual disability;

MRXSSB: Mental retardation, X-linked 102; PMG: Polymicrogyria; WES: Whole

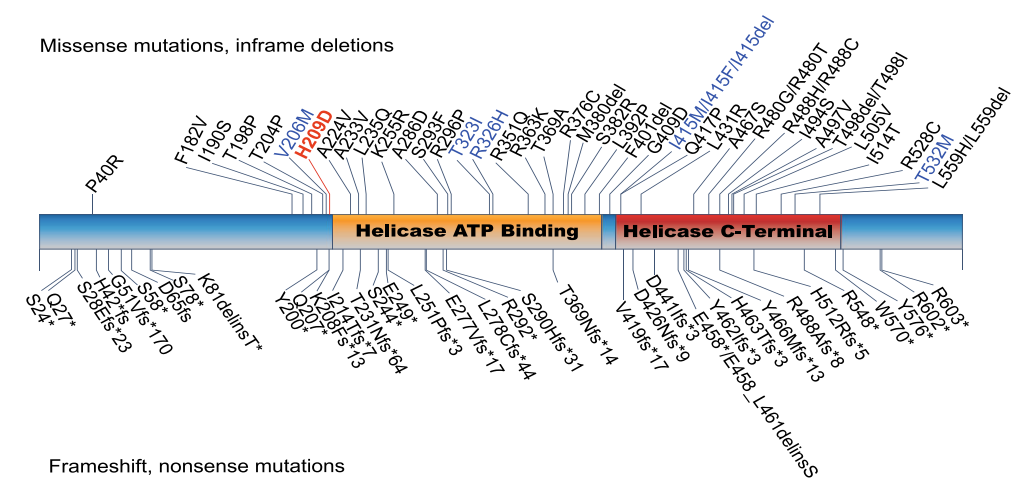

Fig. 2 Schematic representation of the DDX3X protein domains and mapping of mutations already reported in patients affected by the DDX3X syndrome (adapted from [10]). Missense mutations and in-frame deletions are reported on top, while frameshift and nonsense mutations are annotated on the bottom. Mutations reported in patients with PMG are displayed in blue. The His209Asp variant identified in II-3 is reported in red 
exome sequencing; MRI: Magnetic resonance imaging;

EEG: Electroencephalogram; aCGH: Array-Comparative Genomic Hybridization

\section{Supplementary Information}

The online version contains supplementary material available at https://doi. org/10.1186/s13052-021-01033-4

Additional file 1: Supplementary Table 1. Prediction of pathogenicity

of the identified DDX3X variant by in silico tools.

\section{Acknowledgments}

We would like to acknowledge the family for participating in this study and giving consent to publish their data.

Laura Fontana was supported by Fondazione Umberto Veronesi.

\section{Authors' contributions}

GM: WES data analysis and manuscript drafting; JC: bioinformatic analysis; CS: performed WES experiments; OR: WES data analysis and interpretation; FG, EP, SO, DM, PM: clinical evaluation and follow-up of the patient; AP: neuroradiologic analysis; IC: coordinated clinical data collection; LF, MM: supervised the project and contributed to the interpretation of the results. All authors read and approved the final manuscript.

\section{Authors' information}

D.M. is a member of the European Reference Network on Rare Congenital Malformations and Rare Intellectual Disability ERN-ITHACA [EU Framework Partnership Agreement ID: 3HP-HP-FPA ERN-01-2016/739516].

\section{Funding}

This study was supported by Fondazione IRCCS Ca' Granda Ospedale Maggiore Policlinico di Milano (Ricerca Corrente 2019-2020 to MM, Bioinformatics facility for the analysis of omics data and for the design of translational clinical studies).

\section{Availability of data and materials}

The datasets used and/or analyzed during the current study are available from the corresponding author on reasonable request.

\section{Declarations}

\section{Ethics approval and consent to participate}

This work is part of the "CARE: Challenging Approaches to undiagnosed Rare diseases with exome sequencing" Project (Protocol code: PED-CARE-2018).

Appropriate written informed consent was obtained from all family members.

\section{Consent for publication}

Appropriate written informed consent was obtained for publication.

\section{Competing interests}

The authors declare that they have no competing interests.

\begin{abstract}
Author details
${ }^{1}$ Research Laboratories Coordination Unit, Fondazione IRCCS Ca' Granda Ospedale Maggiore Policlinico, Milan, Italy. ${ }^{2}$ Fondazione IRCCS Ca' Granda, Ospedale Maggiore Policlinico, Milan, Italy. ${ }^{3}$ Child Neurology and Psychiatry Unit, IRCCS Mondino Foundation, Pavia, Italy. ${ }^{4}$ Department of Brain and Behavioral Sciences, Università degli Studi di Pavia, Pavia, Italy. ${ }^{5}$ Neuroradiology Department, IRCCS Mondino Foundation, Pavia, Italy. ${ }^{6}$ Department of Pathophysiology and Transplantation, Università degli Studi di Milano, Milan, Italy. ${ }^{7}$ Department of Health Science, Università degli Studi di Milano, Milan, Italy.
\end{abstract}

Received: 23 November 2020 Accepted: 19 March 2021 Published online: 31 March 2021

\section{References}

1. Snijders Blok L, Madsen E, Juusola J, Gilissen C, Baralle D, Reijnders MRF, et al. Mutations in DDX3X are a common cause of unexplained intellectual disability with gender-specific effects on Wnt signaling. Am J Hum Genet. 2015;97(2):343-52. https://doi.org/10.1016/j.ajhg.2015.07.004.

2. The DDX3X Foundation website. https://ddx3x.org/about-ddx3x/. Accessed 24 May 2020

3. Kellaris G, Khan K, Baig SM, Tsai IC, Zamora FM, Ruggieri P, et al. A hypomorphic inherited pathogenic variant in DDX3X causes male intellectual disability with additional neurodevelopmental and neurodegenerative features. Hum Genomics. 2018;12:1-9.

4. Nicola P, Blackburn PR, Rasmussen KJ, Bertsch NL, Klee EW, Hasadsri L, et al. De novo DDX3X missense variants in males appear viable and contribute to syndromic intellectual disability. Am J Med Genet Part A. 2019;179(4):570-8. https://doi.org/10.1002/ajmg.a.61061.

5. Chen CY, Chan CH, Chen CM, Tsai YS, Tsai TY, Lee YHW, et al. Targeted inactivation of murine Ddx3x: essential roles of Ddx3x in placentation and embryogenesis. Hum Mol Genet. 2016;25(14):2905-22. https://doi.org/10.1 093/hmg/ddw143.

6. Garbelli A, Beermann S, Di Cicco G, Dietrich U, Maga G. A motif unique to the human dead-box protein DDX3 is important for nucleic acid binding, ATP hydrolysis, RNA/DNA unwinding and HIV-1 replication. PLoS One. 2011; 6(5):e19810. https://doi.org/10.1371/journal.pone.0019810.

7. Linsalata AE, He F, Malik AM, Glineburg MR, Green KM, Natla S, et al. DDX3X and specific initiation factors modulate FMR 1 repeat-associated non-AUGinitiated translation. EMBO Rep. 2019;20(9):e47498. https://doi.org/10.15252/ embr.201847498.

8. Schröder M. Human DEAD-box protein 3 has multiple functions in gene regulation and cell cycle control and is a prime target for viral manipulation. Biochem Pharmacol. 2010;79(3):297-306. https://doi.org/10.1 016/j.bcp.2009.08.032.

9. Kasim V, Wu S, Taira K, Miyagishi M. Determination of the role of DDX3 a factor involved in mammalian RNAi pathway using an shRNA-expression library. PLoS One. 2013;8(3):e59445. https://doi.org/10.1371/journal.pone. 0059445.

10. Lennox $A L$, Hoye ML, Jiang $R$, Johnson-Kerner BL, Suit LA, Venkataramanan $\mathrm{S}$, et al. Pathogenic DDX3X mutations impair RNA metabolism and neurogenesis during fetal cortical development. Neuron. 2020;106(3):1-17.

11. Beal B, Hayes I, Mcgaughran J, Amor DJ, Miteff C, Jackson V, et al. Expansion of phenotype of DDX3X syndrome: six new cases. Clin Dysmorphol. 2019; 28(4):169-74. https://doi.org/10.1097/MCD.0000000000000289.

12. Phung B, Cieśla M, Sanna A, Guzzi N, Beneventi G, Cao Thi Ngoc P, et al. The X-linked DDX3X RNA helicase dictates translation reprogramming and metastasis in melanoma. Cell Rep. 2019;27:3573-3586.e7.

\section{Publisher's Note}

Springer Nature remains neutral with regard to jurisdictional claims in published maps and institutional affiliations.

Ready to submit your research? Choose BMC and benefit from:

- fast, convenient online submission

- thorough peer review by experienced researchers in your field

- rapid publication on acceptance

- support for research data, including large and complex data types

- gold Open Access which fosters wider collaboration and increased citations

- maximum visibility for your research: over $100 \mathrm{M}$ website views per year

At BMC, research is always in progress.

Learn more biomedcentral.com/submissions 